CLINICAL STUDY

\title{
The prevalence of thyroid disorders during early pregnancy in China: the benefits of universal screening in the first trimester of pregnancy
}

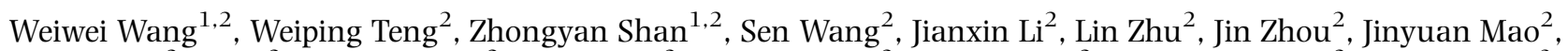 \\ Xiaohui $\mathrm{Yu}^{2}$, Jia Li ${ }^{2}$, Yanyan Chen ${ }^{2}$, Haibo Xue ${ }^{2}$, Chenling $\mathrm{Fan}^{2}$, Hong Wang ${ }^{2}$, Hongmei Zhang ${ }^{2}$, Chenyang $\mathrm{Li}^{3}$, \\ Weiwei Zhou ${ }^{4}$, Bo Gao ${ }^{5}$, Tao Shang ${ }^{6}$, Jiaren Zhou ${ }^{6}$, Bin Ding ${ }^{3}$, Ying $\mathrm{Ma}^{7}$, Ying $\mathrm{Wu}^{8}$, Hui Xu ${ }^{9}$ and Wei Liu ${ }^{10}$ \\ ${ }^{1}$ Department of Endocrinology and Metabolism, The First Hospital of China Medical University, Shenyang, People's Republic of China, 110001 , \\ ${ }^{2}$ The Endocrine Institute of China Medical University, The Liaoning Provincial Key Laboratory of Endocrine diseases, Shenyang, People's Republic of China, \\ 110001, ${ }^{3}$ Shenyang No. 5 People's Hospital, Shenyang, People's Republic of China, 110023, "Women and Infants Hospital of Shenyang, Shenyang, \\ People's Republic of China, 110014, ${ }^{5}$ Shenyang No. 4 People's Hospital, Shenyang, People's Republic of China, 110031, ${ }^{6}$ Second Affiliated Hospital, China \\ Medical University, Shenyang, People's Republic of China, $110004,{ }^{7}$ Shenzhou Hospital of Shenyang, Shenyang, People's Republic of China, 110002, \\ ${ }^{8}$ Shenyang No. 9 People's Hospital, Shenyang, People's Republic of China, 110024, ${ }^{9}$ Women and Infants Hospital of Heping District of Shenyang, \\ Shenyang, People's Republic of China, 110005 and ${ }^{10}$ Shenyang No. 8 People's Hospital, Shenyang, People's Republic of China, 110024 \\ (Correspondence should be addressed to Z Shan; Email: shanzhongyan@medmail.com.cn or to W Teng; Email: twpendocrine@yahoo.com.cn)
}

\begin{abstract}
Context: Maternal thyroid disorders during early pregnancy can influence pregnancy outcome and fetal development. The recent Endocrine Society Clinical Practice Guideline recommends a casefinding approach in which pregnant women who are at high risk for developing thyroid disease are tested.

Objective: The purpose of this study was to use the first trimester-specific reference intervals of thyroidrelated hormones to explore the prevalence of thyroid dysfunction during early pregnancy and to analyze effectiveness of different screening strategies.

Design: A multicenter cohort study.

Method: A total of 2899 pregnant women were enrolled in this study during their first trimester of gestation. Levels of TSH, free thyroxine, free triiodothyronine, and thyroid peroxidase antibodies $(\mathrm{TPOAb})$ were measured and thyroid disorders of pregnant women were diagnosed based on the first trimester-specific reference intervals.

Results: The prevalence of hypothyroidism was significantly higher in the high-risk group than in the non-high-risk group ( 10.9 vs $\left.7.0 \%, \chi^{2}=7.1, P=0.008\right)$. The prevalence of hyperthyroidism was not significantly different between the high-risk group and the non-high-risk group (2.7 vs $1.6 \%$, $\left.\chi^{2}=2.27, P=0.13\right)$. Elevated levels of TPOAb and a personal history of thyroid disease increased the risk of thyroid dysfunction.

Conclusions: A case-finding strategy for screening thyroid function in the high-risk group would miss about $81.6 \%$ pregnant women with hypothyroidism and $80.4 \%$ pregnant women with hyperthyroidism.
\end{abstract}

European Journal of Endocrinology $164263-268$

\section{Introduction}

Development of maternal thyroid disorders during early pregnancy can influence the pregnancy outcome and fetal development. Thyroid dysfunction can lead to premature birth, pregnancy-induced hypertension, increased fetal mortality, and low infant birth weight (1-4). Maternal hypothyroidism and hypothyroxinemia in the first trimester of pregnancy may be harmful to fetal brain development and lead to mental retardation $(5,6)$. In view of the potential adverse outcomes associated with maternal thyroid disorders and the obvious benefits of treatment, some expert panels have suggested routine thyroid function screening in all pregnant women $(7,8)$. However, the Endocrine Society Clinical Practice Guideline (9) recommends a casefinding approach where only women at high-risk for thyroid disorders are tested; these women include those who have a personal or family history of thyroid disease, a personal history of type I diabetes, or other autoimmune disorders, clinical signs suggestive of thyroid disorders, goiter, thyroid antibodies, history of previous therapeutic head or neck irradiation or a history of miscarriage, preterm delivery, or infertility. 
The aim of this study is to use the first trimester-specific reference intervals of thyroid-related hormones to determine the prevalence of thyroid dysfunction during early pregnancy and to evaluate efficiency of the casefinding strategy versus the universal screening strategy.

\section{Materials and methods}

\section{Subjects}

Women were recruited from routine antenatal clinics in ten hospitals from May 2005 through June 2008 in Shenyang, China. Eligibility criteria included pregnant women within the first 12 weeks of gestation and no history of living in endemic goiter areas. A total of 2899 pregnant women living in iodine-adequate areas in their first trimester of pregnancy participated. The medical ethics committee of the First Affiliated Hospital of China Medical University approved this study and all participating women gave informed written consent. Duration of gestation was calculated based on the dates of their last menstrual period and confirmed by ultrasonography. All participants specifically answered a questionnaire about reproductive histories (miscarriages, preterm deliveries, and infertility), personal and family history of thyroid disorders (including first- and second-degree relatives), personal history of type I diabetes or other autoimmune diseases, and history of therapeutic head or neck irradiation by the obstetrician of the ten hospitals. Based on Endocrine Society Clinical Practice Guidelines, women with a personal or family history of thyroid disease, a personal history of type I diabetes or other autoimmune disorders, clinical signs suggestive of thyroid disorders, goiter, thyroid antibodies, a history of previous therapeutic head or neck irradiation, a history of miscarriage, preterm delivery, and infertility were identified as at high risk for thyroid disease during pregnancy (9).

\section{Methods of sampling and laboratory testing}

Blood samples were obtained from each participant in the morning after an overnight fast. All sera obtained were immediately analyzed for TSH, free thyroxine $\left(\mathrm{FT}_{4}\right)$, free triiodothyronine $\left(\mathrm{FT}_{3}\right)$, and thyroid peroxidase antibody $(\mathrm{TPOAb})$ concentrations, using a chemiluminescence immunoassay (Diagnostic Products Corporation, Los Angeles, CA, USA). The functional sensitivity of the TSH assay was $0.02 \mathrm{mIU} / \mathrm{l}$. The intra-assay coefficients of variation $(\mathrm{CV})$ of serum $\mathrm{TSH}, \mathrm{FT}_{4}, \mathrm{FT}_{3}$, TPOAb were $1.57-4.12,2.24-6.33,0.57-4.31$, and $2.42-5.63 \%$ respectively. The inter-assay CV values were $1.26-5.76$, $4.53-8.23,3.50-5.19$, and $5.23-8.16 \%$ respectively. Urinary iodine excretion was measured by the colorimetric ceric ion arsenious acid ash method, based on the Sandell-Kolthoff reaction. The intra- and inter-assay CV values were $<7 \%$.

\section{Trimester-specific reference intervals for TSH, $\mathrm{FT}_{3}$, and $\mathrm{FT}_{4}$ and diagnostic criteria}

The first trimester-specific reference intervals used to diagnose thyroid dysfunctions were based on those determined previously (10). The first trimester-specific reference ranges for TSH, $\mathrm{FT}_{4}$, and $\mathrm{FT}_{3}$ were 0.13-3.93 mIU/l, 12.00-23.34 pM, and 3.46-7.70 pM respectively. Overt hypothyroidism, subclinical hypothyroidism, and hypothyroxinemia were defined by the first trimester-specific reference intervals for TSH, $\mathrm{FT}_{3}$, and $\mathrm{FT}_{4}$ as described previously (10). Overt hyperthyroidism and subclinical hyperthyroidism were identified as decreased TSH levels with increased $\mathrm{FT}_{3}$ or $\mathrm{FT}_{4}$ and with a normal range of $\mathrm{FT}_{3}$ or $\mathrm{FT}_{4}$ respectively. A TPOAb concentration $\geq 50 \mathrm{IU} / \mathrm{ml}$ was considered abnormal.

Women who were found to have thyroid dysfunction or euthyroid women with TPOAb positive had thyroid function tests at least once during the second and third trimesters. Women with subclinical hypothyroidism were recommended to receive $\mathrm{L}-\mathrm{T}_{4}$ treatment and the drug dosage was adjusted according to their serum TSH level. Women with clinical hypothyroidism were offered treatment. Treatment of hyperthyroidism women was based on the endocrinologist's clinical judgment and, if necessary, antithyroid drug was administered.

\section{Statistical analysis}

Data analysis was performed using SPSS software (version 14.5., SPSS, Inc., Cary, NJ, USA). Statistical comparisons were analyzed with the $\chi^{2}$ test. A $P$ value $\leq 0.05$ was considered to be statistically significant. Based on the results of the $\chi^{2}$ test, statistically significant variables were assessed by multivariate logistic regression analysis.

\section{Results}

\section{Characteristics of pregnant women}

The average age of the 2899 women enrolled in the study was $27.61 \pm 3.55$ years old and the median duration of pregnancy at initial analysis was 6 weeks (4-12 weeks). Median urinary iodine of these pregnant women was $177.15 \mu \mathrm{g} / \mathrm{l}$. Fifty-two women $(1.8 \%)$ had personal history of thyroid disorders, which included 21 hypothyroidism (overt or subclinical), 11 hyperthyroidism (overt or subclinical), 11 goiter/thyroid nodule, and 9 other thyroid disorders; 179 women $(6.0 \%)$ had family history of thyroid disorders. Three women $(0.1 \%)$ had history of other autoimmune diseases; 147 women $(5.0 \%)$ had history of miscarriage. Three women $(0.1 \%)$ had history of preterm delivery and five women $(0.2 \%)$ had history of infertility treatment. None had history of head or neck irradiation. Using the Endocrine Society Clinical Practice guidelines (9), we classified $367(12.7 \%)$ women, who had a personal or family 
Table 1 Demographic characteristics of pregnant women.

\begin{tabular}{ll}
\hline Demographic characteristics $(n=2899)$ & \\
\hline Mean maternal age (years) $(x \pm s)$ & $27.61 \pm 3.55$ \\
Median (range) gestational age at & $6(4-12)$ \\
screening (weeks) & \\
Median urinary iodine $(\mu \mathrm{g} / \mathrm{l})$ & 177.15 \\
Number of previous pregnancies, $n(\%)$ & $1492(51.5 \%)$ \\
None & $797(27.5 \%)$ \\
One & $409(14.1 \%)$ \\
Two & $196(6.7 \%)$ \\
Three or more & $5(0.17 \%)$ \\
History of fertility treatment, $n(\%)$ & $147(5.0 \%)$ \\
History of miscarriages, $n(\%)$ & $3(0.1 \%)$ \\
History of preterm delivery, $n(\%)$ & $41(1.4 \%)$ \\
History of smoking, $n(\%)$ & $52(1.8 \%)$ \\
Personal history of thyroid disease, $n(\%)$ & $21(0.7 \%)$ \\
Hyperthyroidism & $11(0.4 \%)$ \\
Hypothyroidism & $11(0.4 \%)$ \\
Goiter/nodule & $9(0.3 \%)$ \\
Other thyroid disease & $0(0.0 \%)$ \\
History of head or neck irradiation, $n(\%)$ & $179(6.2 \%)$ \\
Family history of thyroid disease, $n(\%)$ & $3(0.1 \%)$ \\
Type 1 diabetes/autoimmune & \\
disease ${ }^{\mathrm{a}}, n(\%)$ & \\
\hline anduding rheumatoid arthritis $(n=3)$ &
\end{tabular}

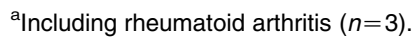

history of thyroid disease, a personal history of type 1 diabetes or other autoimmune disorders, a history of miscarriage, preterm delivery and infertility, a history of head or neck irradiation, as high risk (9). Demographic characteristics of participants are shown in Table 1. There were no significant differences in maternal age, ethnicity, smoking habits, or number of previous pregnancies between the women in the high-risk group and those in the non-high-risk group (data not shown).

\section{Prevalence of thyroid dysfunction in the first trimester of pregnancy and relative risk analysis}

$\mathrm{TSH}, \mathrm{FT}_{4}, \mathrm{FT}_{3}$, and TPOAb levels were measured in all 2899 pregnant women. Of the 2899 women in the study, 294 women had thyroid dysfunction.
The prevalence of thyroid dysfunction was $10.2 \%$. The prevalences of hyperthyroidism, hypothyroidism, and hypothyroxinemia were $1.8,7.5$, and $0.9 \%$ respectively (Table 2). TPOAb was positive in 279 $(9.6 \%)$ of the 2899 women. The number of euthyroid women with antibodies was 196 (6.8\%). The prevalence of thyroid dysfunction in the high-risk group was significantly higher than in non-high-risk group (15.0 vs $\left.9.4 \%, P=0.001, \chi^{2}=10.8\right)$. The prevalence of thyroid dysfunction in pregnant women with personal history of thyroid diseases (30.8 vs $9.8 \%, P=0.000)$, abnormal TPOAb levels (29.7 vs $8.1 \%, P=0.000)$, and personal history of other autoimmune disorders (66.7 vs $10.1 \%, P=0.018$ ) was significantly higher than in pregnant women without the risk factors A logistic multiple regression showed that personal history of thyroid diseases (odds ratio $(\mathrm{OR})=2.3, P=0.016)$ and positive TPOAb $(\mathrm{OR}=4.6, P=0.000)$ were the risk factors for the increase in thyroid dysfunction.

\section{Prevalence of hypothyroidism, hyperthyroidism, and hypothyroxemia in the first trimester of pregnancy and relative risks analysis}

Two hundred and seventeen women (7.5\%) had elevated TSH levels. Eight of them were diagnosed with overt hypothyroidism. Of the eight women with raised $\mathrm{TSH}$ and low $\mathrm{FT}_{4}$, seven had TPOAbs. The prevalence of elevated TSH was higher in the high-risk group than in the non-high-risk group (10.9 vs $7.0 \%$, $\left.\chi^{2}=7.1, P=0.008\right)$. Personal history of thyroid disease $(n=51)$ and the prevalence of hypothyroidism in TPOAb-positive pregnant women $(n=279)$ were significantly higher than those in TPOAb-negative pregnant women $(25.8$ vs $5.5 \%, P=0.000)$ and non-personal history of thyroid disease pregnant women (23.1 vs $7.2 \%, P<0.001)$. It was noteworthy that 177 of 217 women with elevated TSH $(81.6 \%)$ were in the nonhigh-risk group.

Fifty-one women $(1.8 \%)$ with low TSH levels were diagnosed with overt or subclinical hyperthyroidism.

Table 2 The prevalence of thyroid disorders during early pregnancy based on various characteristics. Data are expressed as $n$ (\%).

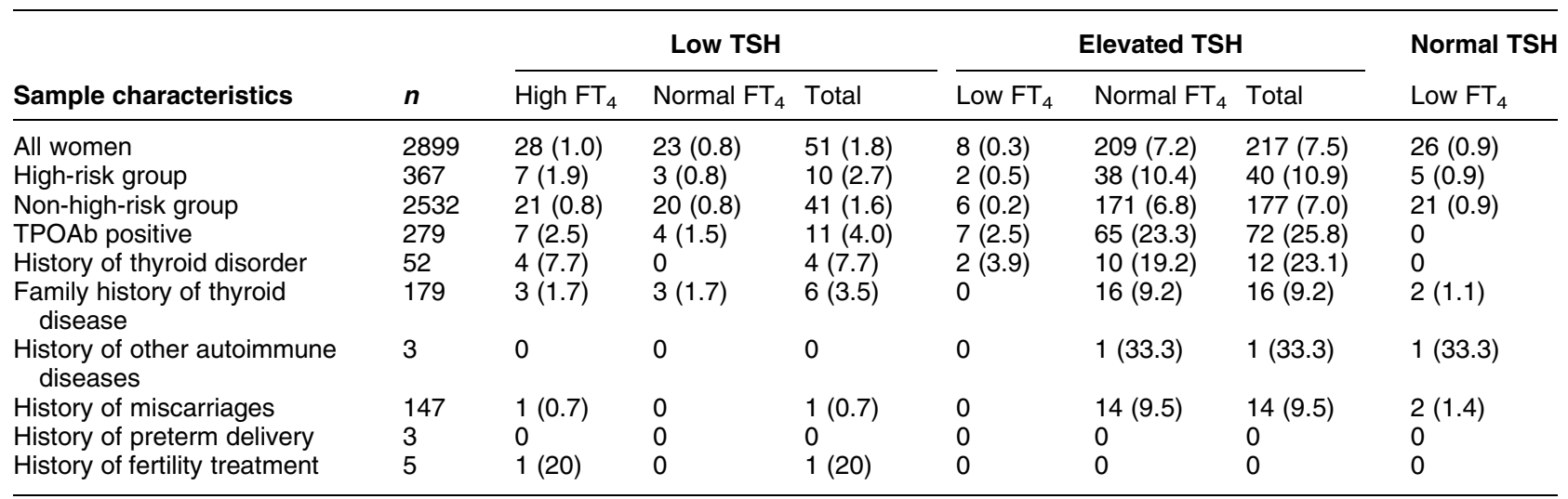




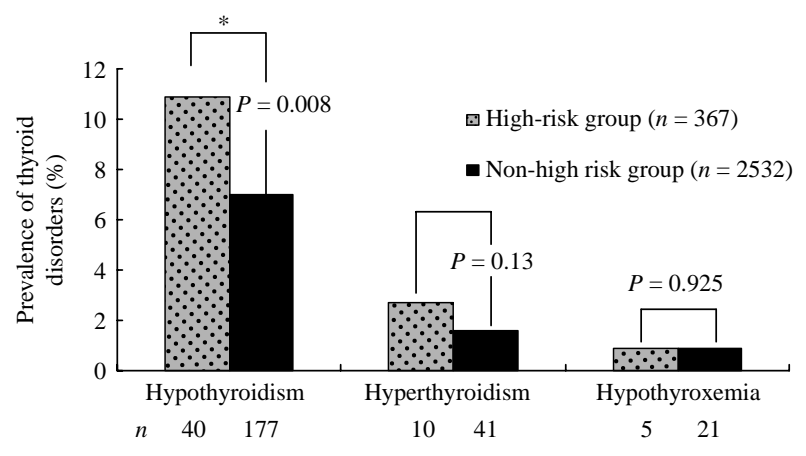

Figure 1 Comparison of the prevalence of thyroid disorders in the high-risk group and the non-high-risk group.

There was no difference in the prevalence of hyperthyroidism between the high-risk group and the nonhigh-risk group $\left(2.7\right.$ vs $\left.1.6 \%, \chi^{2}=2.27, P=0.13\right)$. Personal history of thyroid disease $(n=51)$ and the prevalence of hyperthyroidism in TPOAb-positive pregnant women $(n=279)$ were significantly higher than those in TPOAb-negative pregnant women $(4.0$ vs $1.5 \%, P=0.007)$ and non-personal history of thyroid disease pregnant women ( 7.7 vs $1.7 \%, P=0.006)$. It was noteworthy that only 10 of $51(19.6 \%)$ women with hyperthyroidism belonged to the high-risk group; $41(80.4 \%)$ women with hyperthyroidism were in the non-high-risk group.

There was no difference in the prevalence of hypothyroxemia between the high-risk group and the non-high-risk group (0.9 vs $0.9 \%, \chi^{2}=0.008$, $P=0.928$ ). Figure 1 compares the prevalence of thyroid disorders in the high-risk group and the non-high-risk group and Fig. 2 presents the rates of missed diagnoses if screening were to be performed only in the highrisk population.

A logistic multiple regression was used for risk factor analysis. It showed that positive TPOAb was a risk factor $(\mathrm{OR}=5.8, P=0.000)$ for the increase in hypothyroidism and the presence of TPOAb $(\mathrm{OR}=2.3$, $P=0.022)$ and personal history of thyroid diseases $(\mathrm{OR}=3.6, P=0.025)$ increase the risk of hyperthyroidism (Table 3 ).

\section{Discussion}

Maternal overt hypothyroidism, subclinical hypothyroidism, and hypothyroxinemia are associated with adverse outcomes in pregnancy, including miscarriage, pregnancy-induced hypertension, preterm delivery, placental abruption, and impaired neuropsychological development of children (1-6). Haddow et al. (5) and Pop et al. (6) reported that maternal hypothyroidism and hypothyroxinemia occurring in the first half of pregnancy may be harmful to the embryo-fetal brain development and lead to intellectual retardation in the offspring. Our group also found that maternal subclinical hypothyroidism, hypothyroxinemia, and euthyroidism with elevated TPOAb titers were all statistically significant predictors of lower motor and intellectual development at 25-30 months (11). In Haddow's report, the IQ scores of children whose mothers with hypothyroidism were treated during pregnancy were similar to those of the control children of mothers with normal thyroid function during pregnancy (5). Berbel et al. (12) reported that a delay of 6-10 weeks in iodine supplementation of hypothyroxinemic mothers at the beginning of gestation in an area of mild iodine deficiency increases the risk of neurobehavioral performance delay in their offsprings.

Maternal thyroid function is of great importance for the fetus during the first trimester of pregnancy. Stricker et al. (13) reported that $3.6 \%$ of patients with elevated TSH would be missed and $3.7 \%$ of patients with low TSH level would be misdiagnosed as having a lower TSH level by using the non-pregnant reference intervals to diagnose thyroid diseases. Vaidya et al. (14) concluded that the prevalence of hypothyroxemia increased 3.7\% when the first trimester-specific reference ranges were used rather than general population reference intervals. Our group used two different series of reference intervals to calculate the prevalence of thyroid hormone deficiency and also found that $2 \%$ patients with subclinical hypothyroidism would be misclassified if general population reference intervals were used (10). These results indicate that the first trimester-specific reference ranges must be used to evaluate thyroid function in pregnancy.

In this study, we evaluated the prevalence of thyroid dysfunction by screening 2899 pregnant women using the first trimester-specific reference intervals from our previous work (10). The results showed that high-risk women, classified based on Endocrine Society Clinical Practice Guidelines (9), had more than a 1.5-fold increased risk of hypothyroidism (subclinical or overt) and a 1.7-fold increased risk of hyperthyroidism (subclinical or overt) during early pregnancy than did women in the non-high-risk group. However, screening for thyroid diseases only in the high-risk pregnant women, as the guidelines recommend, would have meant that about $81.6 \%$ women with hypothyroidism $(2.7 \%$ with overt hypothyroidism and $78.8 \%$ with

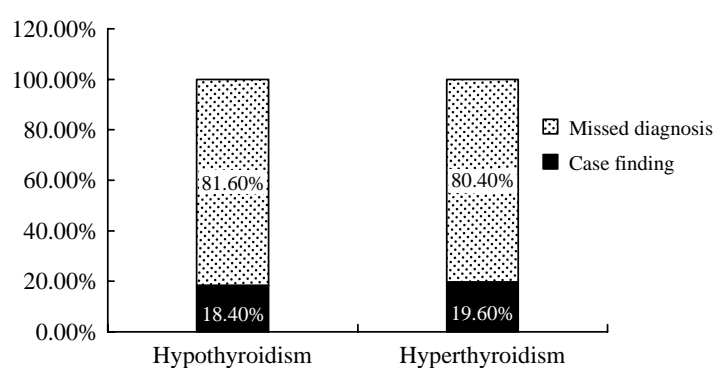

Figure 2 The rates of missed diagnoses if screening were to be performed only in the high-risk population. 
Table 3 Relative risks for hypothyroidism and hyperthyroidism at screening.

\begin{tabular}{|c|c|c|c|c|c|c|}
\hline \multirow[b]{2}{*}{ Risk factors } & \multicolumn{3}{|c|}{ Hypothyroidism } & \multicolumn{3}{|c|}{ Hyperthyroidism } \\
\hline & OR & $95 \% \mathrm{Cl}$ & $P$ & OR & $95 \% \mathrm{Cl}$ & $P$ \\
\hline TPOAb positive & 5.8 & $4.2-8.1$ & 0.000 & 2.3 & $1.1-4.7$ & 0.022 \\
\hline Personal history of thyroid disorder & 2.0 & $1.0-4.2$ & 0.057 & 3.6 & $1.2-10.9$ & 0.025 \\
\hline Family history of thyroid disorders & 1.0 & $0.6-1.8$ & 0.879 & 1.9 & $0.8-4.5$ & 0.172 \\
\hline $\begin{array}{l}\text { Personal history of other } \\
\text { autoimmune disorders }\end{array}$ & 3.5 & $0.3-44.6$ & 0.342 & 0.0 & 0.0 & 0.999 \\
\hline History of miscarriage & 1.2 & $0.7-2.2$ & 0.584 & 0.2 & $0.3-1.8$ & 0.169 \\
\hline History of preterm delivery & 0.0 & 0.0 & 0.999 & 0.0 & 0.0 & 0.999 \\
\hline History of fertility treatment & 0.0 & 0.0 & 0.999 & 17.0 & $1.9-155.2$ & 0.012 \\
\hline
\end{tabular}

subclinical hypothyroidism) and $80.4 \%$ women with hyperthyroidism $(41.2 \%$ with overt hyperthyroidism and $39.2 \%$ with subclinical hyperthyroidism) would be missed. Our results were similar to those reported by Vaidya et al. (14), who concluded that targeted thyroid function testing of only high-risk pregnant women would miss nearly one third of women with overt/ subclinical hypothyroidism during early pregnancy by using non-pregnant population reference intervals. A higher percentage of missed diagnoses occured in this study than in the Vaidya study maybe because Vaidya defined euthyroidism in that study as a TSH $<4.2$, whereas this study defined euthyroidism as a TSH $<3.93$. Recently, another study (15) reached similar conclusions. In that study, pregnant women were randomized in the first trimester to either universal screening group or case-finding group. The study showed that the case-finding approach fails to detect the majority of pregnant women with thyroid dysfunction (15). The benefits and risks of universal testing or case-finding strategies in pregnant women are debated (16-19). As Brent (8) had previously pointed out that the potential adverse outcomes are so significant and the tools to diagnose and treat thyroid disease are easily accessible, that it is no longer acceptable for even one third of pregnant women to remain undiagnosed. Our results support performing universal screening early in pregnancy for thyroid disorders using the first trimester-specific reference intervals. In 2008, Dosiou et al. (20) reported that screening pregnant women for TSH concentrations in the first trimester of pregnancy was cost saving compared with no screening, and screening using anti-TPO antibodies was also economically favorable. The study of Thung used marginal cost per quality-adjusted life year as the main outcome measure and show that universal screening for subclinical hypothyroidism in pregnancy would be a cost-effective strategy (21). Regarding the main argument against universal screening has been the lack of clinical trial evidence showing that treatment for mild maternal hypothyroidism prevents neuropsychological deficit in children and other obstetric complications and also that more randomized trials are needed.

The most appropriate screening test for thyroid dysfunction in early pregnancy is still uncertain. Most would advocate using TSH as the initial screening test, because TSH is a more sensitive marker of thyroid status than $\mathrm{FT}_{4}$ and it reflects the physiologic log/linear relationship of TSH to $\mathrm{FT}_{4}(22,23)$. TPOAb measurement should also be considered due to the relationship with adverse pregnancy events, such as post partum thyroiditis, recurrent miscarriage, and post partum depression. In addition, $20-30 \%$ of women with post partum thyroiditis develop permanent hypothyroidism $(24,25)$. Negro et al. reported that treatment of euthyroid pregnant women who were positive for TPOAb with $\mathrm{L}_{-} \mathrm{T}_{4}$ lowered the chance of miscarriage and premature delivery. Selenium supplementation during pregnancy and in the post partum period also reduces thyroid inflammatory activity and the incidence of post partum thyroid dysfunction and hypothyroidism $(26,27)$. Maternal hypothyroxinemia during pregnancy may affect neuropsychological development of offspring $(6,11,12)$, which affected $0.9 \%$ of the cohort of this study with equal frequency in the high-risk and non-high-risk groups, and is identified only if serum TSH is measured. Therefore, we support using TSH, $\mathrm{FT}_{4}$, and TPOAb as the initial screening test for thyroid dysfunction in early pregnancy.

In conclusion, our study confirms that a case-finding strategy for screening thyroid function would miss about $81.6 \%$ pregnant women with hypothyroidism and $80.4 \%$ pregnant women with hyperthyroidism.

\section{Declaration of interest}

The authors declare that there is no conflict of interest that could be perceived as prejudicing the impartiality of the research reported.

\section{Funding}

The study was supported by the National Public Welfare Foundation, the Healthcare Special Project (grant numbers 200802008), and the Social Development Foundation in Liaoning Province (grant numbers 2007225010), all in China.

\section{Acknowledgements}

We are indebted to the residents of Shenyang who participated in this study and gratefully acknowledge the help of doctors from ten hospitals of Shenyang. 


\section{References}

1 Allan WC, Haddow JE, Palomaki GE, Williams JR, Mitchell ML, Hermos RJ, Faix JD \& Klein RZ. Maternal thyroid deficiency and pregnancy complications: implications for population screening. Journal of Medical Screening 20007 127-130. (doi:10.1136/jms.7. 3.127)

2 Casey BM \& Leveno KJ. Thyroid disease in pregnancy. Obstetrics and Gynecology 2006108 1283-1292. (doi:10.1097/01.AOG. 0000244103.91597.c5)

3 Casey BM, Dashe JS, Wells CE, McIntire DD, Byrd EW, Leveno KJ \& Cunningham FG. Subclinical hypothyroidism and pregnancy outcomes. Obstetrics and Gynecology 2005105 239-245. (doi:10.1097/01.AOG.0000152345.99421.22)

4 Abalovich M, Gutierrex S, Alcaraz G, Maccallini G, Garcia A \& Levalle 0 . Overt and subclinical hypothyroidism complicating pregnancy. Thyroid 200212 63-66. (doi:10.1089/10507250 2753451986)

5 Haddow JE, Palomaki GE, Allan WC, Williams JR, Knight GJ, Gagnon J, O'Heir CE, Mitchell ML, Hermos RJ, Waisbren SE, Faix JD \& Klein RZ. Maternal thyroid deficiency during pregnancy and subsequent neuropsychological development of the child. New England Journal of Medicine 1999341 549-555. (doi:10. 1056/NEJM199908193410801)

6 Pop VJ, Brouwers EP, Vader HL, Vulsma T, van Baar AL \& de Vijlder JJ. Maternal hypothyroxinaemia during early pregnancy and subsequent child development: a 3-year follow-up study. Clinical Endocrinology 200359 282-288. (doi:10.1046/j.13652265.2003.01822.x)

7 Gharib H, Tuttle RM, Baskin J, Fish LH, Singer PA \& McDermott MT. Subclinical thyroid dysfunction: a joint statement on management from the American Association of Clinical Endocrinologists, the American Thyroid Association, and The Endocrine Society. Thyroid 200515 24-28. (doi:10.1089/thy. 2005.15.24)

8 Brent GA. Diagnosing thyroid dysfunction in pregnant women: is case finding enough? Journal of Clinical Endocrinology and Metabolism 200792 39-41. (doi:10.1210/jc.2006-2461)

9 Abalovich M, Amino N, Barbour LA, Cobin RH, De Groot LJ, Glinoer D, Mandel SJ \& Stagnaro-Green A. Management of thyroid dysfunction during pregnancy and postpartum: an Endocrine Society clinical practice guideline. Journal of Clinical Endocrinology and Metabolism 200792 (Suppl) S1-S47. (doi:10.1210/jc.2007-0141)

10 Shan ZY, Chen YY \& Teng WP. A study for maternal thyroid hormone deficiency during the first half of pregnancy in China. European Journal of Clinical Investigation 200939 37-42. (doi:10. 1111/j.1365-2362.2008.02055.x)

11 Li Y, Shan Z, Teng W, Yu X, Li Y, Fan C, Teng X, Guo R, Wang H, Li J, Chen Y, Wang W, Chawinga M, Zhang L, Yang L, Zhao Y \& Hua T. Abnormalities of maternal thyroid function during pregnancy affect neuropsychological development of their children at 25-30 months. Clinical Endocrinology 201072 825-829. (doi:10.1111/j. 1365-2265.2009.03743.x)

12 Berbel P, Mestre JL, Santamaría A, Palazón I, Franco A, Graells M, González-Torga A \& de Escobar GM. Delayed neurobehavioral development in children born to pregnant women with mild hypothyroxinemia during the first month of gestation: the importance of early iodine supplementation. Thyroid $2009 \mathbf{1 9}$ 511-519. (doi:10.1089/thy.2008.0341)

13 Stricker RT, Echenard M \& Eberhart R. Evaluation of maternal thyroid function during pregnancy: the importance of using gestational age-specific reference intervals. European Journal of Endocrinology 2007157 509-514. (doi:10.1530/EJE-07-0249)

14 Vaidya B, Anthony S, Bilous M, Shields B, Drury J, Hutchison S \& Bilous R. Detection of thyroid dysfunction in early pregnancy: universal screening or targeted high-risk case finding. Journal of Clinical Endocrinology and Metabolism 200792 203-207. (doi:10. 1210/jc.2006-1748)
15 Negro R, Schwartz A, Gismondi R, Tinelli A, Mangieri T \& Stagnaro-Green A. Universal screening versus case finding for detection and treatment of thyroid hormonal dysfunction during pregnancy. Journal of Clinical Endocrinology and Metabolism 2010 95 1699-1707. (doi:10.1210/jc.2009-2009)

16 American College of Obstetricians and Gynecologists. ACOG Practice Bulletin. Clinical management guidelines for obstetrician-gynecologists. Number 37, August 2002 (Replaces Practice Bulletin Number 32, November 2001). Thyroid disease in pregnancy. Obstetrics and Gynecology 2002100 387-396. (doi:10.1016/S0029-7844(02)02196-8)

17 Surks MI, Ortiz E, Daniels GH, Sawin CT, Col NF, Colin RH, Franklyn JA, Hershman JM, Burman KD, Denke MA, Gorman C, Cooper RS \& Weissman NJ. Subclinical thyroid disease: scientific review and guidelines for diagnosis and management. Journal of the American Medical Association 2004291 228-238. (doi:10. 1001/jama.291.2.228)

18 Hollowell JG, LaFranchi S, Smallridge RC, Spong CY, Haddow JE \& Boyle CA. Where do we go from here? Summary of working group discussions on thyroid function and gestational outcomes Thyroid 200515 72-76. (doi:10.1089/thy.2005.15.72)

19 American Thyroid Association. Consensus Statement \#2: American Thyroid Association statement on early maternal thyroidal insufficiency: recognition, clinical management and research directions. Thyroid 200515 77-79. (doi:10.1089/thy. 2005.15.77)

20 Dosiou C, Sanders GD, Araki SS \& Crapo LM. Screening pregnant women for autoimmune thyroid disease: a cost-effectiveness analysis. European Journal of Endocrinology 2008158 841-851. (doi:10.1530/EJE-07-0882)

21 Thung SF, Funai EF \& Grobman WA. The cost-effectiveness of universal screening in pregnancy for subclinical hypothyroidism. American Journal of Obstetrics and Gynecology 2009200 267.e1-267.e7. (doi:10.1016/j.ajog.2008.10.035)

22 Dashe JS, Casey BM, Wells CE, McIntire DD, Byrd EW, Leveno KJ \& Cunningham FG. Thyroid-stimulating hormone in singleton and twin pregnancy: importance of gestational age-specific reference ranges. Obstetrics and Gynecology 2005106 753-757. (doi:10. 1097/01.AOG.0000175836.41390.73)

23 Mandel SJ, Spencer CA \& Hollowell JG. Are detection and treatment of thyroid insufficiency in pregnancy feasible? Thyroid 200515 44-53. (doi:10.1089/thy.2005.15.44)

24 Premawardhana LD, Parkes AB \& Ammari F. Postpartum thyroiditis and long-term thyroid status: prognostic influence of thyroid peroxidase antibodies and ultrasound echogenicity. Journal of Clinical Endocrinology and Metabolism 200085 71-75. (doi:10.1210/jc.85.1.71)

25 Pop VJ, De Rooy HA \& Vader HL. Postpartum thyroid dysfunction and depression in an unselected population. New England Journal of Medicine 1991324 1815-1816. (doi:10.1056/NEJM19910620 3242516)

26 Negro R, Greco G, Mangieri T, Pezzarossa A, Dazzi D \& Hassan H. The influence of selenium supplementation on postpartum thyroid status in pregnant women with thyroid peroxidase autoantibodies. Journal of Clinical Endocrinology and Metabolism 200792 1263-1268. (doi:10.1210/jc.2006-1821)

27 Negro R, Formoso G, Mangieri T, Pezzarossa A, Sazzi D \& Hassan H. Levothyroxine treatment in euthyroid pregnant women with autoimmune thyroid disease: effects on obstetrical complications. Journal of Clinical Endocrinology and Metabolism 200691 2587-2591. (doi:10.1210/jc.2005-1603)

Received 26 October 2010

Accepted 8 November 2010 